\title{
Vascular Imaging as a Cardiovascular Risk Stratification Tool in Systemic Lupus Erythematosus
}

Systemic lupus erythematosus (SLE) is an autoimmune disorder associated with increased incidence of cardiovascular disease (CVD) and consequently, a higher rate of cardiovascular events ${ }^{1}$. This association has been mainly established on epidemiological data and, given that patients with SLE are predominantly premenopausal women, this excess in CVD risk might appear somewhat unexpected ${ }^{1,2}$.

Fifty years ago, only $50 \%$ of patients with SLE survived 5 years after diagnosis; but today, with better therapeutic strategies, the disease outcome is better, and $80 \%$ to $90 \%$ of patients survive at least 10 years after SLE is diagnosed ${ }^{3}$. With the increased life expectancy of patients with SLE, CVD has perhaps emerged as a more significant threat to their health. For a long time, the effect of this problem has been under-recognized, with little focus on aggressive management of CVD risk factors or the development of risk stratification strategies.

\section{BIMODAL MORTALITY PATTERN: THE FACTS}

The high risk for CVD in patients with SLE was substantiated by studies that assessed the incidence of major cardiovascular events and studies that noninvasively evaluated the atherosclerotic burden in SLE. Almost 3 decades ago, Urowitz, et al followed up 81 SLE patients for 5 years and reported that in the majority of those who died late in the course of the disease, myocardial infarction was the primary cause of death ${ }^{3}$. These authors first described a phenomenon that is still known as the "bimodal mortality pattern" of SLE.

Recent prospective studies have also confirmed this unusual bimodal pattern of mortality in SLE. Manzi, et al ${ }^{4}$ reported that women with SLE at the age of 35 to 44 years were over 50 times more likely to have a myocardial infarction than women of similar age in the Framingham Offspring Study. The authors concluded that CVD was much more common in young premenopausal women with SLE compared to a random population sample ${ }^{4}$. Similarly, a case-control study from the British General Practice Research Database showed an increased risk of myocardial infarction in patients with SLE even after adjustment for conventional cardiovascular risk factors, history of CVD, and renal failure ${ }^{5}$. Of note, longterm followup (from 1958 to 2001) of a large international cohort of 9547 patients with SLE revealed a slight increase in cardiovascular mortality over the last 3 decades, in contrast to the observed overall decline in mortality and mortality from septic shock and renal failure ${ }^{6}$. Thus, the immediate question that follows is why the cardiovascular system of SLE patients would appear to be so vulnerable?

\section{HOW IS ATHEROSCLEROSIS LINKED TO SLE?}

There is probably no single and comprehensive answer on this question. The factors promoting atherosclerosis in SLE are complex and are still not completely understood. An association between systemic vasculitis present in SLE and coronary artery disease has been proposed. However, autopsy studies have demonstrated that the coronary vessels of SLE patients have the typical atherosclerotic plaques, and that most cardiovascular events are not attributable to active vasculitis ${ }^{7}$. Another theory suggests that systemic inflammation related to SLE contributes to plaque destabilization. Indeed, acute coronary syndromes are caused by acute disruption of unstable plaques, and the process of plaque destabilization is intimately linked to the upregulation of inflammatory cytokines and the consequent endothelial activation that occurs more commonly in SLE ${ }^{8}$.

Additionally, it has been suggested that prevalence of CVD risk factors is more common in patients with SLE either due to the disease itself or as a result of treatment for the disease. Indeed, altered lipid profiles and impaired glucose tolerance have been reported in patients with SLE, and their burden correlates well with their disease activity ${ }^{9,10}$. Further, glucocorticoid therapy might also promote the development of atherosclerosis by altering lipid and glucose metabolism, as well as by elevating blood pressure levels ${ }^{11}$. However, no clear correlation has been found between use of corticosteroid therapy and atherosclerosis in SLE. Indeed, there are even studies reporting an increase in cardiovascular risk among patients who are under-treated with corticosteroids, and perhaps inadequate disease control

See Pattern of arterial calicification in SLE, page 2212

Personal non-commercial use only. The Journal of Rheumatology Copyright @ 2009 . All rights reserved. 
may confer a higher vascular risk compared to the administration of glucocorticoid therapy per $\mathrm{se}^{12}$. Admittedly, atherosclerosis itself is progressively recognized as a systemic inflammatory process involving the vascular wall. Thus, one may speculate that chronic SLE-related inflammation may represent a major factor leading to plaque development and destabilization.

\section{NONINVASIVE ASSESSMENT OF ATHEROSCLER- OTIC BURDEN IN SLE PATIENTS: THE FUTURE}

Despite the gaps in our understanding of the underlying pathophysiology, it is well established that SLE patients are at increased risk of cardiovascular events. Thus, it is essential to institute efficient strategies for the identification and management of patients at increased risk. However, application of standard risk stratification tools in SLE patients may be problematic.

For example, several studies have demonstrated that the Framingham risk equation performs poorly in patients with $\mathrm{SLE}^{13}$. The Framingham equation is used to predict the 10 -year risk of myocardial infarction, and is strongly based on age and sex, but validated in the general population although ignoring special subsets of patients such as those with connective tissue diseases ${ }^{13}$. Moreover, conventional risk factors of CVD fail to reliably predict the cardiovascular risk associated with $\mathrm{SLE}^{2}$. Thus, it is essential to find other ways to efficiently identify SLE patients at increased risk for CVD - perhaps a potential way to do so is by noninvasive screening for the presence of subclinical atherosclerosis.

At present, there are several methods of identifying subclinical atherosclerosis. For example, single-photon emission-computed tomographic myocardial perfusion imaging is one method, and in one study, $40 \%$ of 130 SLE patients studied had perfusion abnormalities that suggested subclinical coronary artery disease ${ }^{14}$. Reversible defects consistent with ischemia constituted $90.4 \%$ of the abnormalities seen. In $27 \%$ of patients fixed defects were identified consistent with previous myocardial necrosis, and these defects were seen in the distribution of the epicardial coronary vessel supply rather than diffuse abnormalities suggestive of small-vessel disease ${ }^{14}$.

Vascular ultrasonography has also been used in assessing the atherosclerotic burden of SLE. For example, Roman, et $a l^{12}$ recently reported data comparing the prevalence of carotid plaque (detected using B-mode ultrasound) in a cohort of women with SLE compared with a control population, and SLE patients had a significantly higher prevalence of significant carotid plaques. Similar findings were reported by Ahmad, et $a l^{15}$ in a study of 200 women with SLE and 100 controls.

Noninvasive testing of endothelial dysfunction is another popular way of screening for subclinical atherosclerosis, given that endothelial dysfunction is associated with atherosclerotic vascular disease. For example, El Magadmi, et al $l^{16}$ reported impairment of endothelial function estimated using flow-mediated dilatation in SLE patients ${ }^{16}$.

Conventional echocardiography is useful to assess cardiac structure and function, but is unable to estimate the atherosclerotic burden per $\mathrm{se}^{17}$. Nevertheless, new echocardiographic indexes, contrast agents, and imaging software have increased the potential of this technique. Thus, it is possible now to evaluate coronary flow reserve by transthoracic echocardiography in patients with systemic autoimmune disease, in order to detect abnormalities in myocardial perfusion. More recently, other imaging modalities including magnetic resonance and computed tomography (CT) have allowed imaging of the coronary arteries. Arterial calcification assessed either by electron beam CT or multidetector CT (MDCT) has been evaluated in SLE patients, and its presence correlates with disease duration, presence of CVD risk factors, and exposure to corticosteroids ${ }^{18}$.

In this issue of The Journal, Yiu, et al ${ }^{19}$ applied 64-slice MDCT scanning to evaluate calcium scores (CS) in coronary and carotid arteries, as well as the aorta of SLE patients. The authors report that compared with controls, patients with SLE had higher mean CS and higher prevalence of CS $>0$ across all vascular beds. In SLE patients, the most frequent vessel with CS $>0$ was the coronary artery $(42 \%)$ followed by the carotid artery $(24 \%)$. Further, arterial calcification occurred early, involving $40 \%$ of SLE patients aged $<40$ years old, with increasing prevalence as age advanced.

Calcium deposition along the coronary arteries is reasonably good evidence of atherosclerosis. High coronary artery CS correlates well with advanced disease and a higher likelihood of coronary stenoses, and numerous studies have supported the role of CS as a screening tool for coronary artery disease. Indeed, comparative studies with intravascular ultrasound and histology have demonstrated the capability of MDCT to provide information that is useful for characterizing the atherosclerotic plaque in a noninvasive manner ${ }^{20}$. However, despite evidence suggesting a strong negative predictive value of MDCT assessed CS in the general population (especially in the subset of low-risk patients) there are relatively limited data regarding special populations such as patients with connective tissue disease. Thus, the article by Yiu, et $a l^{19}$ extends the field, by demonstrating, in a well designed prospective case-control study, that patients with SLE have high prevalence and extended vascular calcification detected by MDCT.

Nonetheless, calcification of the vascular wall is a nonspecific process closely related to atherogenesis. Thus, the cynic would argue that the study contributes little in our understanding of the precise mechanisms underlying the increased prevalence of atherosclerosis among patients with SLE. However, the article by Yiu, et $a l^{19}$ provides indirect evidence suggesting that in a specific subset of patients, CS is a potent tool for the identification of high-risk individuals.

Personal non-commercial use only. The Journal of Rheumatology Copyright @ 2009 . All rights reserved. 


\section{WHERE DO WE GO FROM HERE?}

Prospective studies are clearly required to validate the importance of CS in CVD risk stratification of patients with SLE. Also, longitudinal studies with disease-modifying agents, or drugs that have vasculoprotective benefits (e.g., statins), to show their effects on CS in patients with SLE would help define the utility of this vascular "marker." Valid prognostic information obtained by these new cardiac imaging techniques will perhaps help risk stratification and aid our management of patients with SLE, to improve their outcome.

\section{STAVROS APOSTOLAKIS, MD; EDUARD SHANTSILA, MD; GREGORY Y.H. LIP, MD,}

Haemostasis Thrombosis and Vascular Biology Unit, University of Birmingham Centre for Cardiovascular Sciences, City Hospital, Birmingham, UK

Address correspondence to Prof. Lip. E-mail: g.y.h.lip@bham.ac.uk

\section{REFERENCES}

1. Soubrier M, Mathieu S, Dubost JJ. Atheroma and systemic lupus erythematosus. Joint Bone Spine 2007;74:566-70.

2. Bruce IN. 'Not only...but also': factors that contribute to accelerated atherosclerosis and premature coronary heart disease in systemic lupus erythematosus. Rheumatology 2005;44:1492-502.

3. Urowitz MB, Bookman AA, Koehler BE, Gordon DA, Smythe HA, Ogryzlo MA. The bimodal mortality pattern of systemic lupus erythematosus. Am J Med 1976;60:221-5.

4. Manzi S, Meilahn EN, Rairie JE, Conte CG, Medsger TA Jr, Jansen-McWilliams L, et al. Age-specific incidence rates of myocardial infarction and angina in women with systemic lupus erythematosus: comparison with the Framingham Study. Am J Epidemiol 1997;145:408-15.

5. Fischer LM, Schlienger RG, Matter C, Jick H, Meier CR. Effect of rheumatoid arthritis or systemic lupus erythematosus on the risk of first-time acute myocardial infarction. Am J Cardiol 2004;93:198-200.

6. Bernatsky S, Boivin JF, Joseph L, Manzi S, Ginzler E, Gladman $\mathrm{DD}$, et al. Mortality in systemic lupus erythematosus. Arthritis Rheum 2006;54:2550-7.

7. Haider YS, Roberts WC. Coronary arterial disease in systemic lupus erythematosus; quantification of degrees of narrowing in 22 necropsy patients (21 women) aged 16 to 37 years. Am J Med $1981 ; 70: 775-81$.
8. Selzer F, Sutton-Tyrrell K, Fitzgerald SG, Pratt JE, Tracy RP, Kuller LH, et al. Comparison of risk factors for vascular disease in the carotid artery and aorta in women with systemic lupus erythematosus. Arthritis Rheum 2004;50:151-9.

9. Borba EF, Bonfa E. Dyslipoproteinemias in systemic lupus erythematosus: influence of disease, activity, and anticardiolipin antibodies. Lupus 1997;6:533-9.

10. El Magadmi M, Ahmad Y, Turkie W, Yates AP, Sheikh N, Bernstein RM, et al. Hyperinsulinemia, insulin resistance, and circulating oxidized low density lipoprotein in women with systemic lupus erythematosus. J Rheumatol 2006;33:50-6.

11. MacGregor AJ, Dhillon VB, Binder A, Forte CA, Knight BC, Betteridge DJ, et al. Fasting lipids and anticardiolipin antibodies as risk factors for vascular disease in systemic lupus erythematosus. Ann Rheum Dis 1992;51:152-5.

12. Roman MJ, Shanker BA, Davis A, Lockshin MD, Sammaritano L, Simantov R, et al. Prevalence and correlates of accelerated atherosclerosis in systemic lupus erythematosus. N Engl J Med 2003;349:2399-406.

13. Chung CP, Oeser A, Avalos I, Gebretsadik T, Shintani A, Raggi P, et al. Utility of the Framingham risk score to predict the presence of coronary atherosclerosis in patients with rheumatoid arthritis. Arthritis Res Ther 2006;8:R186.

14. Nikpour M, Gladman DD, Ibañez D, Bruce IN, Burns RJ, Urowitz MB. Myocardial perfusion imaging in assessing risk of coronary events in patients with systemic lupus erythematosus. J Rheumatol 2009;36:288-94.

15. Ahmad Y, Bodill H, Shelmerdine J, Bernstein RM, Walker MG, Teh L, et al. Antiphospholipid antibodies (APLA) contribute to atherogenesis in SLE [abstract]. Arthritis Rheum 2004;50 Suppl:S191.

16. El Magadmi M, Bodill H, Ahmad Y, Durrington PN, Mackness M, Walker M, et al. Systemic lupus erythematosus: an independent risk factor for endothelial dysfunction in women. Circulation 2004;110:399-404.

17. Turiel M, Peretti R, Sarzi-Puttini P, Atzeni F, Doria A. Cardiac imaging techniques in systemic autoimmune diseases. Lupus 2005;14:727-31.

18. Kiani AN, Magder L, Petri M. Coronary calcium in systemic lupus erythematosus is associated with traditional cardiovascular risk factors, but not with disease activity. J Rheumatol 2008;35:1300-6.

19. Yiu KH, Wang S, Mok MY, Ooi GC, Khong PL, Mak KH, et al. Pattern of arterial calcification in patients with systemic lupus erythematosus. J Rheumatol 2009;36:2212-7.

20. Marano R, Bonomo L. Coronary artery calcium score: has anything changed? Radiol Med 2007;112:949-58.

J Rheumatol 2009;36:2141-3; doi:10.3899/jrheum.090640 